\title{
Valproate $v$. lithium in the treatment of bipolar disorder in clinical practice: observational nationwide register-based cohort study
}

\author{
Lars Vedel Kessing, Gunnar Hellmund, John R. Geddes, Guy M. Goodwin and Per Kragh Andersen
}

\section{Background}

Valproate is one of the most used mood stabilisers for bipolar disorder, although the evidence for the effectiveness of valproate is sparse.

\section{Aims \\ To compare the effect of valproate $v$. lithium for treatment of bipolar disorder in clinical practice.}

\section{Method}

An observational cohort study with linkage of nationwide registers of all people with a diagnosis of bipolar disorder in psychiatric hospital settings who were prescribed valproate or lithium in Denmark during a period from 1995 to 2006.

\section{Results}

A total of 4268 participants were included among whom 719 received valproate and 3549 received lithium subsequent to the diagnosis of bipolar disorder. The rate of switch/add on to the opposite drug (lithium or valproate), antidepressants, antipsychotics or anticonvulsants (other than valproate) was increased for valproate compared with lithium (hazard ratio $(\mathrm{HR})=1.86,95 \% \mathrm{Cl}$ 1.59-2.16). The rate of psychiatric hospital admissions was increased for valproate $v$. lithium $(H R=1.33$, $95 \% \mathrm{Cl} 1.18-1.48)$ and regardless of the type of episode leading to a hospital admission (depressive or manic/mixed) Similarly, for participants with a depressive index episode $(\mathrm{HR}=1.87,95 \% \mathrm{Cl} 1.40-2.48)$, a manic index episode
$(H R=1.24,95 \% \mathrm{Cl} 1.01-1.51)$ and a mixed index episode $(H R=1.44,95 \% \mathrm{Cl} 1.04-2.01)$, the overall rate of hospital admissions was significantly increased for valproate compared with lithium.

\section{Conclusions}

In daily clinical practice, treatment with lithium seems in general to be superior to treatment with valproate.

\section{Declaration of interest}

L.V.K. has been a consultant for Bristol-Myers Squibb, Eli Lilly, Lundbeck, Astrazenica, Pfizer, Wyeth, Servier, Janssen-Cilag. J.R.G. has received research funding from the Medical Research Council (MRC), Economic and Social Research Council, National Institute for Health Research, and the Stanley Medical Research Institute, and has received donations of drugs supplies for trials from Sanofi-aventis and GlaxoSmithKline. He has acted as an expert witness for Dr Reddys. G.M.G. has received research grants from Baily Thomas, MRC, Sanofi-aventis, and Servier; honoraria from AstraZeneca, Bristol-Myers Squibb, Eisai, Lundbeck, Sanofiaventis, and Servier; is a shareholder in P1vital; has a paid position at University of Oxford; and has been a member of advisory boards with AstraZeneca, Bristol-Myers Squibb, Lilly, Janssen-Cilag, Lundbeck, P1Vital, Sanofi-aventis, Servier, Roche, and Wyeth. He has acted as an expert witness for Lilly and Servier.
Bipolar disorder is a complex heterogeneous recurrent episodic illness with frequent comorbidity, high suicidality and requiring complex pharmacological treatment. Valproate is one of the most commonly used mood stabilisers worldwide. Indeed, in the USA, valproate is the most used mood stabiliser given in monotherapy followed by lithium, ${ }^{1}$ although the evidence for its effectiveness remains somewhat sparse. Valproate has been best investigated in the treatment of acute mania where it was superior to placebo in three trials ${ }^{2-4}$ but not significantly different from lithium in two out of three studies, ${ }^{3,5}$ lithium being slightly more efficacious overall in the third study. ${ }^{6,7}$ Valproate has been found to be effective in acute depression in three out of four small placebocontrolled trials, of which the largest included 54 participants, and two are unpublished. ${ }^{8}$ No study of bipolar depression has compared valproate with lithium. Finally, two maintenance studies on valproate have been published. In the only placebocontrolled monotherapy maintenance study, neither valproate nor lithium showed clear prophylactic effect compared with placebo during 1-year follow-up ${ }^{9}$ and in the recent 2-year BALANCE study lithium was more effective than valproate ${ }^{10}$ in a mixed population, some of whom were also receiving other long-term medicines.

In a recent statement, the US National Institute of Health committed itself to comparative effectiveness research and large scale observational studies. ${ }^{11}$ Although randomised controlled trials (RCTs) are fundamental for demonstrating the efficacy and safety of medications, their applicability to people with bipolar disorder may be complicated by the complex and labile symptomatic presentations of the illness, a tendency for individuals to deny illness or reject treatment, and diagnostic heterogeneity in routine practice. ${ }^{12}$ Clinicians frequently note that the effectiveness of medication may be substantially less than the efficacy estimated from highly controlled randomised data selectively submitted to regulatory agencies. ${ }^{13}$ Further, realistically, only a small number of large-scale RCTs will ever be conducted compared with the number of important questions, for example, the cost of the Clinical Antipsychotic Trials of Intervention Effectiveness Study (CATIE) was over $\$ 40$ million. ${ }^{14}$ Thus, evidence from randomised trials may be usefully supplemented by data from large-scale observational cohort studies that typically include a wide and unselected range of individuals with a high treatment variability. ${ }^{15,16}$

We present data from a population-based and nationwide register linkage study on all individuals with a diagnosis of bipolar disorder within psychiatric hospital healthcare settings who were treated with valproate or lithium during a study period of up to 12 years. We compared both rates of switch to, or add on of, another psychotropic drug and psychiatric hospital admissions for the two drugs. 


\section{Method}

\section{Danish register data}

Data were obtained by linking Danish population-based registers using the unique personal identification number (CPR-number), which is assigned to all 5.3 million people living in Denmark, thus ensuring accurate linkage of information between registers, irrespective of changes in name, etc. ${ }^{17}$ In this way, the Danish Psychiatric Central Register ${ }^{18}$ was linked with the Medicinal Product Statistics ${ }^{19}$ and the Danish Medical Register on Vital Statistics. ${ }^{20}$

The Danish Psychiatric Central Register ${ }^{18}$ contains data on all in-patients from 1970 and data on all out-patients treated at all psychiatric hospitals in Denmark from 1 January 1995 and onwards as a part of the official Danish health survey. ${ }^{21}$ Since 1 January 1994 the ICD-10 has been in use in the register. ${ }^{22}$ Thus, in recent decades, data are available about accurate numbers of hospital admissions.

The Medicinal Product Statistics contains data on all prescribed medication purchased at pharmacies from 1 January 1995 and onwards. ${ }^{19}$ In Denmark, all medication prescribed by doctors, such as lithium and anticonvulsants, is purchased only at pharmacies and the following data are electronically recorded in the Medicinal Product Statistics: the CPR-number of the individual, the ATC code of the drug (Anatomical Therapeutical Chemical classification system ${ }^{23}$ ), and the dose and the number of tablets of the drug. Medication given during a hospital admission is not recorded in the register. Neither the daily dosing of the medication nor the indication for treatment are registered. The Danish Medical Register on Vital Statistics ${ }^{20}$ contains data about deaths.

\section{Study sample}

We identified all individuals who received for the first time a main ICD-10 index diagnosis of bipolar disorder (ICD-10 code: F30-31.9 + 38.00) at contact with a psychiatric in-patient or out-patient service during the study period from 1 January 1995 to 31 December 2006 and who, following the index diagnosis, purchased lithium (N05AN, N05AN01) or valproate (N03AG01) at least once. From this population we excluded all individuals who prior to their index diagnosis of bipolar disorder had purchased lithium or valproate. Thus, the majority of participants included in the study had been prescribed lithium or valproate previously but a minority may have been given the drugs before the study window opened.

\section{Statistical analyses}

In the analyses with switch to or add on as the outcome measure, participants who purchased antidepressants, antipsychotics or anticonvulsants (other than valproate) at the same date as they purchased lithium or valproate were excluded. Cox regression analyses were conducted comparing the rates of switch to or add on of another psychotropic drug for participants who purchased either lithium or valproate as monotherapy (besides benzodiazepines and drugs for somatic illnesses), censoring at a diagnosis of schizophrenia (ICD-10 code: F20-20.9), death and end of the study period (31 December 2006). Another psychotropic drug was defined as the opposite drug of interest (lithium or valproate), antidepressants, antipsychotics or anticonvulsants (other than valproate).

In the analyses with psychiatric hospital admissions as the outcome the total study sample as defined above was included. Cox regression analyses were conducted comparing the rates of hospital admissions for participants who purchased lithium and those who purchased valproate, censoring at purchase of the opposite drug of interest (individuals who initially purchased lithium were censored at the date of purchasing valproate, and vice versa), a diagnosis of schizophrenia, death and at end of the study period.

Analyses were adjusted for gender (male/female), age group in 5 -year intervals (up to 24 years, $25-29,30-34$, etc. up to $60-64$, $65+$ ), years since diagnosis $(0,1,2$, etc. up to 11$)$, purchase of antidepressants, antipsychotics or anticonvulsants (other than valproate) prior to purchase of lithium or valproate, and previous number of psychiatric hospital admissions. In the analyses with hospital admissions as the outcome, the models were further adjusted dynamically for the time-dependent covariates of concomitant use of antidepressants (yes, no), antipsychotics (yes, no) and anticonvulsants (other than valproate, yes, no).

The analyses were carried out further for four subpopulations:

(a) participants with an index diagnosis of bipolar disorder, current episode depression (ICD-10 codes: F31.3-31.51);

(b) participants with a diagnosis of a hypomanic or manic episode or bipolar disorder, current episode hypomanic or manic (ICD-10 codes: F30-31.21);

(c) participants with a mixed episode or bipolar disorder, current episode mixed (ICD-10 codes: F31.6 or 38.00); and

(d) participants with bipolar disorder, currently in remission (ICD-10 code: F31.7).

When psychiatric hospital admissions was the outcome, analyses were made with overall hospital admissions as the outcome and then with hospital admissions with a depressive episode and those with a manic/mixed episode as respective outcomes.

Cumulative incidence curves for the risks of interest were estimated for the total population accounting for the competing risks.

\section{Results}

A total of 11404 people were recorded in the Danish Psychiatric Central Register with a main diagnosis of bipolar disorder between 1995 and 2006 inclusive and who had not previously received a diagnosis of schizophrenia. Among this population, the following individuals were excluded: 2584 people who had purchased lithium prior to the index diagnosis, 160 who had purchased valproate prior to the diagnosis, 98 who had purchased both lithium and valproate prior to index diagnosis and 32 who purchased lithium and valproate simultaneously following the index diagnosis. Among the remaining 8562 individuals, a total of 4268 purchased either lithium $(n=3549,83.2 \%)$ or valproate $(n=719,16.8 \%)$ subsequent to the bipolar index diagnosis, whereas 25 people were excluded because of a diagnosis of schizophrenia between index diagnosis and first purchase, 9 people had an event on day of first purchase and 4228 individuals did not purchase any of these drugs. The population of 4268 participants treated with either lithium or valproate was analysed with psychiatric hospital admissions as the outcome (Table 1). Gender distributions and age at first purchase of lithium and valproate can be seen to be indistinguishable for the full population and for specific subgroups by index episode (depressive, manic, mixed, remission). The date of index contact for a bipolar diagnosis was the date of discharge from a psychiatric in-patient hospital admission for $67 \%$ of those participants who were subsequently prescribed lithium and for $69 \%$ of those prescribed valproate. In the remaining population, medication 
was prescribed following the date of the first psychiatric out-patient index contact. The numbers of psychiatric hospital admissions (including the index hospital admission) prior to treatment was also similar for those prescribed lithium (median 2, interquartile range $(\mathrm{IQR})=1-5)$ and those prescribed valproate (median 3, IQR $=1-6$ ). Prior to the index episode the proportions of participants receiving other medications were: for antidepressants $69 \%$ of those taking lithium and $75 \%$ of those taking valproate; for antipsychotics $54 \%$ of those taking lithium and $73 \%$ of those taking valproate; and other anticonvulsants $20 \%$ of those taking lithium and $34 \%$ for of those taking valproate. The median duration from the index diagnosis of bipolar disorder to first purchase of valproate was 0.2 years $(\mathrm{IQR}=0.0-1.6)$ and to first purchase of lithium it was 0.1 years $(\mathrm{IQR}=0.0-0.7)$. The use of medication concomitant with lithium/valproate during follow-up was as follows: $63 \%$ of those taking lithium and $60 \%$ of those taking valproate used antidepressants; $59 \%$ of those taking lithium and $75 \%$ of those taking valproate used antipsychotics and $17 \%$ of those taking lithium and $13 \%$ of those taking valproate used anticonvulsants (other than valproate). All Cox regression models were adjusted dynamically for this time-dependent concomitant use. A total of 266 participants died during follow-up, 214 (6\%) in the lithium group and $52(7 \%)$ in the valproate group, and 135 participants switched to the opposite drug. Table 1 presents the rate ratios of psychiatric hospital admissions for all 4268 participants, and then by subgroup according to the different index episodes of bipolar disorder. There were high rates of readmission overall and in the diagnostic subgroups.

For the total population, the overall hazard rate for psychiatric hospital admissions was significantly increased for valproate $v$. lithium $(\mathrm{HR}=1.33,95 \%$ CI 1.18-1.48). This was seen for the rate of hospital admissions with a depressive episode ( $\mathrm{HR}=1.32,95 \%$ CI 1.17-1.58) as well as for hospital admissions for a manic/mixed episode (HR $=1.29,95 \%$ CI 1.13-1.47).

For different index episodes, the largest effects favouring lithium were evident for index depression ( $\mathrm{HR}=1.87,95 \% \mathrm{CI}$ 1.40-2.48); effects after manic $(\mathrm{HR}=1.24,95 \%$ CI 1.01-1.51) and mixed index episodes ( $\mathrm{HR}=1.44,95 \%$ CI 1.04-2.01) were more equivocal (Table 1). For participants in remission, the overall rate of hospital admissions did not differ between the drugs. Additionally, the rates of hospital admissions with a depressive episode and the rate with a manic/mixed episode were significantly increased for valproate compared with lithium for individuals with a manic as well as for people with a mixed index episode, but not for those with a depressive index episode or for individuals in remission (Table 1). Figure 1 illustrates the time course of psychiatric hospital admissions for participants treated with lithium or valproate, with increasing divergence favouring lithium after about 1 year of treatment. The cumulative incidence of hospital admissions, as presented in the figure, is uncorrected for any effect of other variables.

Analyses using switch to/add on in the smaller population of 1845 individuals included 1555 (84.3\%) who purchased lithium and $290(15.7 \%)$ who purchased valproate. Table 2 presents characteristics and rate ratios of switch to or add on of another psychotropic in all participants with bipolar disorder, and the subgroup of participants with different index episodes, and treated with valproate $v$. lithium in monotherapy. Characteristics such as age, gender, history of psychiatric hospital admissions and drug treatment were very similar to those for the 4268 participants included in the analyses of hospital admissions. The hazard rate of switch to or add on of another psychotropic drug (i.e. the opposite drug of interest (lithium or valproate), antidepressants, antipsychotics or anticonvulsants (other than valproate)) was

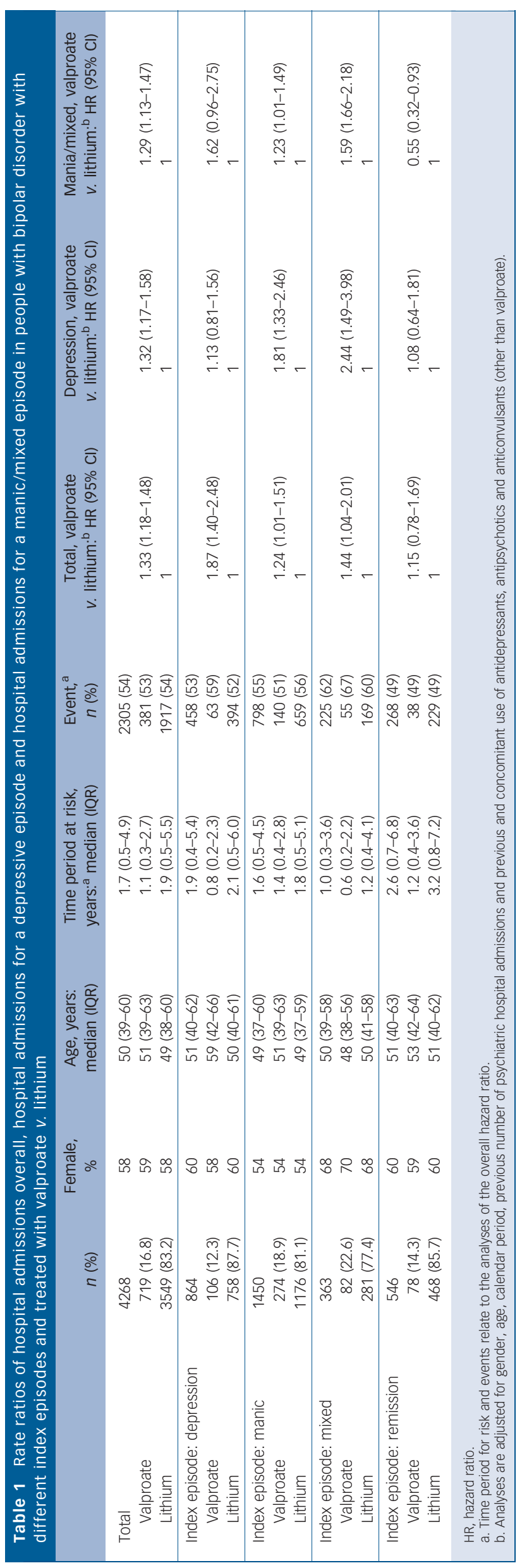




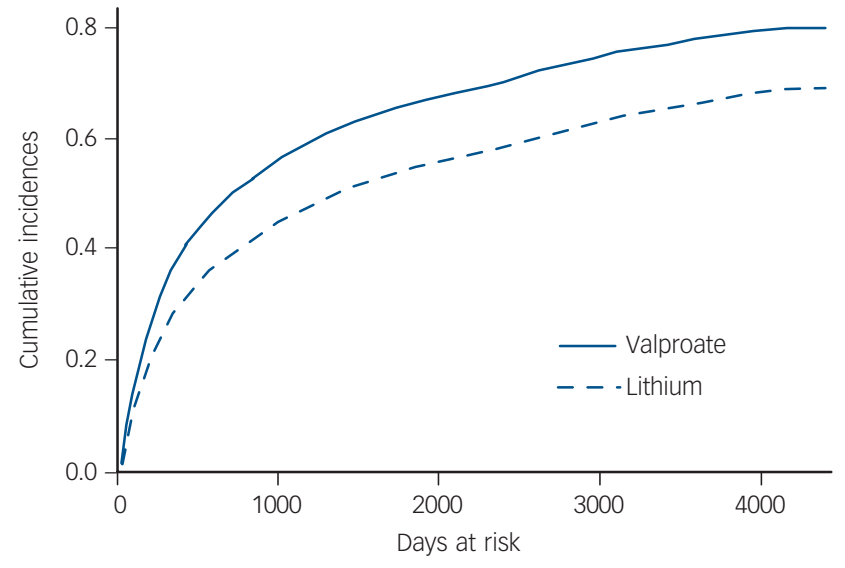

Fig. 1 Cumulative incidence curves for the risk of psychiatric hospital admissions in people with bipolar disorder treated with valproate $v$. lithium (uncorrected).

significantly increased for valproate compared with lithium, and for the subgroup with a depressive or a manic index episode. The largest effects favouring lithium was evident for index depression $(\mathrm{HR}=2.52,95 \% \mathrm{CI} 1.72-3.70)$. For individuals with a mixed index episode and for those in remission, differences between the drugs did not reach statistical significance. Figure 2 illustrates the time course of switch to/add on for participants treated with lithium or valproate, with increasing divergence favouring lithium from the first months of starting treatment. As with Fig.1, the cumulative incidence of switch to/add on, as presented in the figure, is uncorrected for any effect of other variables. It should be noted that differences between the two drugs in terms of the cumulative risks of switch to/add on (Fig. 2) may partly be the result of early intolerability for both drugs with a higher rate of switch to/add on for valproate, as the main difference between the two curves emerged during the first few months of treatment.

\section{Discussion}

This population-based, nationwide register linkage study included up to 12 years follow-up and is by far the largest study, with the

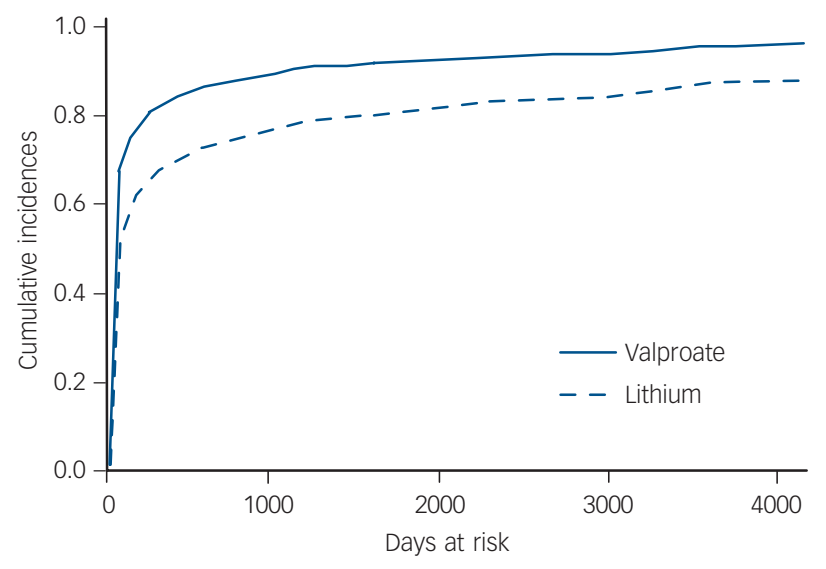

Fig. 2 Cumulative incidence curves for the risk of switch to or add on of another psychotropic ${ }^{a}$ in people with bipolar disorder treated with valproate $v$. lithium in monotherapy (uncorrected).

a. Another psychotropic: the opposite drug of interest (lithium or valproate), antidepressants, antipsychotics or anticonvulsants (other than valproate).

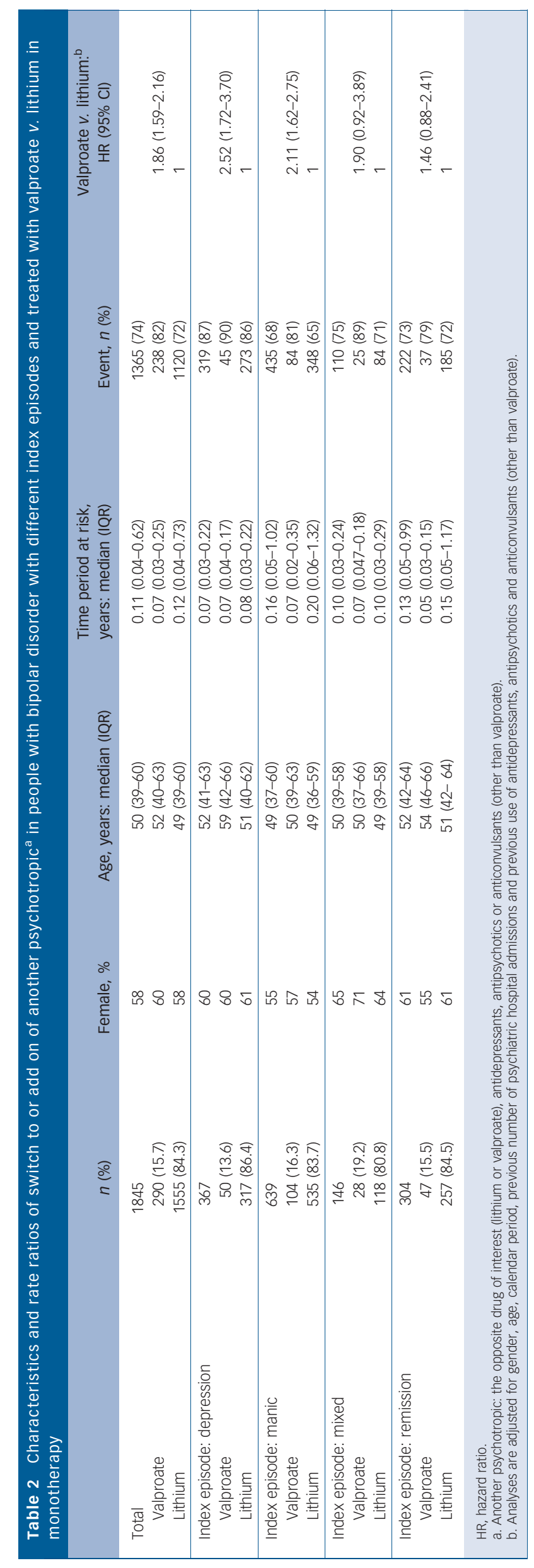


longest follow-up, of individuals treated with valproate or lithium ever described. The overall rate of psychiatric hospital admissions was increased for people treated with valproate compared with lithium after about 1 year, most marked for those with an index episode of depression. The overall rate of switch/add on to the opposite drug (lithium or valproate), antidepressants, antipsychotics or anticonvulsants (other than valproate) was increased for valproate compared with lithium as was the rate for people with a depressive index episode and the rate for those with a manic index episode. In summary, treatment with lithium was in general and overall superior to treatment with valproate.

The study is naturalistic in nature and design, so that participants were not allocated treatment at random and outcomes may be subject to confounding factors. We have minimised the potential impact of earlier treatment by selecting only participants receiving lithium or valproate for the first time. Hospital admissions are an important hard outcome almost always indicating significant treatment failure. When individuals start with valproate or lithium as monotherapy, addition of another drug indicates that the initial drug was not sufficient to improve affective symptoms or maintain remission, or in the case of a switch, there was an inadequate response or intolerable side-effects.

Lithium is frequently used in Denmark. A total of $0.3 \%$ of the Danish population was treated with lithium during a period from 1995 to 1999 inclusive ${ }^{24}$ compared with $0.06 \%$ of the German population $^{25}$ (we are not aware of other published national prevalence rates for lithium). Reasons for this relatively frequent use may be that lithium was introduced early in Denmark due to the pioneer work of Mogens Schou ${ }^{26,27}$ and for decades it has been regarded as the drug of choice of all the mood stabilisers. ${ }^{28}$ Nevertheless, the frequency of lithium treatment does not reach the recommended level of $0.6 \%$ of the population as suggested by Müller-Oerlinghausen et al as a rational treatment strategy. ${ }^{25}$ In the present study, $16.5 \%$ of participants were treated with valproate in monotherapy and $83.5 \%$ with lithium in monotherapy (Table 1). We chose to compare valproate with lithium because lithium is the most used active comparator in randomised trials for bipolar disorder. Lithium has, for more than 60 years, been the main mood-stabilising treatment for bipolar disorder with good evidence. ${ }^{29,30}$ Nevertheless, for some individuals the use of lithium may be hampered by side-effects and a lack of benefit. Further, the evidence for an effect on acute depression ${ }^{8}$ and mania $^{31}$ is weaker.

\section{Strengths and weaknesses}

The diagnoses in the Danish Psychiatric Central Register originate from different clinicians all over Denmark using ICD-10 criteria for bipolar disorder and are not standardised for research purposes. The ICD-10 criteria for bipolar disorder includes bipolar I as well as bipolar II disorders but does not discriminate between these two. Data from the Medicinal Product Statistics is close to $100 \%$ accurate, as drugs prescribed by doctors cannot be purchased at pharmacies without individualised data being electronically submitted to the Medicinal Product Statistics.

Results from RCTs often have limited generalisability to clinical practice as these trials usually have strict inclusion and exclusion criteria resulting in highly selected samples of participants with bipolar disorder who usually do not have comorbidities, such as alcohol, cannabis or substance misuse, or who suffer from less severe illnesses because individuals with psychosis, rapid cycling, substance misuse/dependence or those at high risk of suicide are frequently excluded. ${ }^{13,16}$ As a result of these inclusion and exclusion criteria, RCTs within affective disorders include selected populations of between 15 and 35\% of patients seen in clinical practice. ${ }^{32-34}$ The present study includes all individuals in Denmark who had a diagnosis of bipolar disorder given to them in psychiatric hospital care settings as in-patients and who subsequently were treated with valproate or lithium as out-patients, as well as all out-patients in psychiatric hospital care settings without a prior hospital admission. All treatment data were included for all out-patient settings, i.e. within psychiatric hospital out-patients, community psychiatric centres, private specialist practice and general practice. Individuals treated in psychiatric hospital care settings more often experience severe, complicated and treatment-resistant bipolar disorder. Thus, participants included in the analyses may have been treated with drugs other than valproate or lithium by general practitioners or private psychiatrists prior to contact with a psychiatric hospital service, as might be reflected in the relatively old sample (median age 50 years, $I Q R=39-60$ ). In this way, our findings may not be directly applicable to people who are diagnosed with milder forms of bipolar disorder in general practice or in private psychiatric practice and who do not gain access to psychiatric hospital care, or to those who have undiagnosed bipolar disorder. The background study population of individuals with bipolar disorder from which the samples for analyses were drawn included 11404 people treated in psychiatric hospital care settings, corresponding to $0.3 \%$ of the approximately 4 million adults in Denmark.

We identified people with a diagnosis of bipolar disorder who received lithium or valproate for the first time by excluding 2482 $(21.8 \%)$ individuals who were given these drugs before their bipolar disorder diagnosis. Nevertheless, a minority of individuals included in the analyses may not have been incident users of lithium or valproate as they may have got these drugs before the start of the observation period in 1995 . We find it unlikely that such inclusion of some prevalent users has biased our findings, as there are no indications in the literature that the effect of incident $v$. prevalent use of the drugs should differ systematically between lithium and valproate.

Potential confounding effects are the major fundamental problems in the interpretation of data from cohort studies. As data were non-randomised, they reflect associations in clinical practice in which doctors choose to prescribe valproate and lithium based on a combination of their knowledge and clinical experience with effects and side-effects of the drugs, their clinical evaluation of the illness of the individual involved and the patient preferences. Valproate was relatively more often prescribed for a manic $(18.9 \%)$ or a mixed $(22.6 \%)$ index episode than for a depressive episode $(12.3 \%)$ or during remission (14.3\%, Table 1). In this way, treatment practice in Denmark may reflect the better evidence for valproate in the treatment of manic/mixed episodes than for a depressive episode or as maintenance therapy. Age, gender and number of prior psychiatric hospital admissions were quite similar for participants prescribed valproate and those prescribed lithium, but individuals who were prescribed valproate more often had been previously treated with antidepressants, antipsychotics and anticonvulsants (other than valproate) and participants who were prescribed lithium were more often prescribed concomitant medication with antidepressants and anticonvulsants (other than valproate) but less often antipsychotics (Tables 1 and 2 and Results section). We cannot exclude the possibility that individuals who were prescribed valproate may have been a harder-to-treat population, although we do not find any clear differences between the valproate- and lithium-treated populations. Further, we took account of the effect of such differences, including differences in prior and concomitant treatment, by adjusting all analyses for these variables. The statistical models with hospital admissions as the outcome were 
adjusted dynamically for time-dependent concomitant use of antipsychotics, antidepressants and anticonvulsants, so it not likely that participants on lithium did better in relation to hospital admissions because of adjunctive treatment. It should be acknowledged, nevertheless, that an effect of residual or unknown confounders cannot be neglected. All analyses in the study were further adjusted for the effect of years since diagnosis - in this way also taking account of any changes in the healthcare system during the study period.

The register contains no data on the prescribed dose of the drugs. Both valproate and lithium serum levels are routinely monitored according to standardised clinical practice. Although the relationship between response and lithium serum levels is not entirely clear, ${ }^{35}$ it cannot be excluded that lithium serum levels were monitored more frequently than valproate levels because of the lack of evidence for a therapeutic range in bipolar disorder for valproate.

We did not include analyses on individuals treated with combined valproate and lithium as such analyses would be confounded by indication, since people who receive combined treatment most likely experience more severe disorders and thus will have a poorer outcome due to the non-randomised nature of our data.

\section{Comparison with prior studies}

Our findings are similar to the findings in the randomised openlabel BALANCE trial that found that lithium monotherapy compared with valproate monotherapy resulted in decreased intervention for an emergent mood episode, including change in the drug treatment or admission to hospital $(\mathrm{HR}=0.71,95 \% \mathrm{CI}$ $0.51-1.00) .{ }^{10}$ As in the present study, these effects were seen early for switch to/add on and after 1 year for admission to hospital. One of the strengths of the BALANCE study is that participants were randomly allocated to treatment group irrespective of any previous response to the study drugs. This is in contrast to the enriched design used in most trials of long-term therapy in bipolar disorder. ${ }^{36}$ This design helps to increase the generalisability of the results as they apply to bipolar disorder irrespective of the index episode prior to randomisation. In addition, the wide range of individuals that were enrolled from various locations and clinical situations further ensures that the findings are broadly applicable to people with bipolar I disorder. ${ }^{10}$ On the other hand, a number of individuals were not included in the randomised phase of the study as $28 \%$ (129 out of 459 ) of participants withdrew from the 4-8 weeks run-in phase with treatment with both lithium and valproate; further, the study report presented no data on non-participants not wanting to be enrolled in a randomised trial. However, the similar findings in the BALANCE study and our naturalistic study suggest that the results from the BALANCE study can be generalised to all people with bipolar disorder in psychiatric hospital settings as in-patients or out-patients.

Lithium has been associated with a decreased rate of suicide in randomised studies ${ }^{37}$ and in observational studies (compared with valproate $^{38}$ or to anticonvulsants in general $\left.{ }^{24,39}\right)$, further demonstrating the power of concordant results from randomised and non-randomised data.

In addition, as a result of the large data-set we were able to present findings according to different index mood episodes; however, the number of some events limited the statistical power of some secondary analyses. We found that when the index episode was manic (or mixed), treatment with valproate was associated with an increased rate of switch to/add on as well as an increased rate of subsequent hospital admissions overall and for depressive and manic/mixed relapses. These findings are in accordance with results from an RCT on acute mania showing decreased efficacy of valproate $v$. lithium in rates of response/ remission ${ }^{6}$ but in contrast to other studies showing similar efficacy of the two drugs. ${ }^{3,5}$ No RCT has compared valproate and lithium for treatment of bipolar depression but our results show that when the index episode was depressive, treatment with valproate was overall associated with increased rates of switch to/add on and hospital admissions when compared with lithium, but the rates of hospital admissions for subtypes of episodes did not differ.

\section{Implications}

In daily clinical practice, treatment with lithium is in general superior to treatment with valproate and the advantage of lithium is most apparent following treatment for a depressive episode. We believe that these data provide important supplemental evidence that adds to results from RCTs on valproate $v$. lithium. It is remarkable that after so long lithium remains the most effective treatment as monotherapy or in combination for the treatment of bipolar disorder.

Lars Vedel Kessing, MD, DMSc, Psychiatric Center Copenhagen, University Hospital of Copenhagen, Rigshospitalet, Denmark; Gunnar Hellmund, MSC, PhD, Department of Biostatistics, University of Copenhagen, Copenhagen, Denmark; John R. Geddes, MD, Guy M. Goodwin MD PhD, Department of Psychiatry, University of Oxford, Warneford Hospital, Oxford, UK; Per Kragh Andersen, MSC, PhD, DMSC, Department of Biostatistics, University of Copenhagen, Copenhagen, Denmark

Correspondence: Lars Vedel Kessing, Psychiatric Center Copenhagen, University Hospital of Copenhagen, Rigshospitalet, Blegdamsvej 9, 2100 Copenhagen, Denmark. Email: lars.vedel.kessing@regionh.dk

First received 14 Jul 2010, final revision 19 Jan 2011, accepted 23 Feb 2011

\section{References}

1 Baldessarini R, Henk H, Sklar A, Chang J, Leahy L. Psychotropic medications for patients with bipolar disorder in the United States: polytherapy and adherence. Psychiatr Serv 2008; 59: 1175-83.

2 Pope Jr HG, McElroy SL, Keck Jr PE, Hudson JI. Valproate in the treatment of acute mania. A placebo-controlled study. Arch Gen Psychiatry 1991; 48: $62-8$.

3 Bowden $\mathrm{CL}$, Brugger AM, Swann AC, Calabrese JR, Janicak PG, Petty F, et al. Efficacy of divalproex vs lithium and placebo in the treatment of mania. The Depakote Mania Study Group. JAMA 1994; 271: 918-24.

4 Bowden CL, Swann AC, Calabrese JR, Rubenfaer LM, Wozniak PJ, Collins MA, et al. A randomized, placebo-controlled, multicenter study of divalproex sodium extended release in the treatment of acute mania. $J$ Clin Psychiatry 2006; 67: 1501-10.

5 Bowden C, Gogus A, Grunze H, Haggstrom L, Rybakowski J, Vieta E. A 12-week, open, randomized trial comparing sodium valproate to lithium in patients with bipolar I disorder suffering from a manic episode. Int Clin Psychopharmacol 2008; 23: 254-62.

6 Freeman TW, Clothier JL, Pazzaglia P, Lesem MD, Swann AC. A double-blind comparison of valproate and lithium in the treatment of acute mania. Am J Psychiatry 1992; 149: 108-11.

7 Macritchie K, Geddes JR, Scott J, Haslam D, de LM, Goodwin G. Valproate for acute mood episodes in bipolar disorder. Cochrane Database Syst Rev 2003; 1: CD004052.

8 Grunze H, Vieta E, Goodwin GM, Bowden C, Licht RW, Möller HJ, et al. The World Federation of Societies of Biological Psychiatry (WFSBP) Guidelines for the Biological Treatment of Bipolar Disorders: Update 2010 on the treatment of acute bipolar depression. World J Biol Psychiatry 2010; 11 81-109.

9 Bowden CL, Calabrese JR, McElroy SL, Gyulai L, Wassef A, Petty F, et al. A randomized, placebo-controlled 12-month trial of divalproex and lithium in treatment of outpatients with bipolar I disorder. Divalproex Maintenance Study Group. Arch Gen Psychiatry 2000; 57: 481-9.

10 Geddes JR, Goodwin GM, Rendell J, Azorin JM, Cipriani A, Ostacher MJ, et al. Lithium plus valproate combination therapy versus monotherapy for relapse prevention in bipolar I disorder (BALANCE): a randomised open-label trial. Lancet 2010; 375: 385-95. 
11 Lauer MS, Collins FS. Using science to improve the nation's health system: NIH's commitment to comparative effectiveness research. JAMA 2010; 303 2182-3.

12 Baldessarini RJ. Treatment research in bipolar disorder: issues and recommendations. CNS Drugs 2002; 16: 721-9.

13 Calabrese JR, Kemp DE. Bipolar drug development: are we getting closer to the real world? Am J Psychiatry 2008; 165: 1234-6.

14 Citrome L. Interpreting and applying the CATIE results: with CATIE, context is key when sorting out Phases 1, 1A, 1B, 2E, and 2T. Psychiatry 2007; 4: 23-9.

15 Licht RW. Limitations in randomised controlled trials evaluating drug effects in mania. Eur Arch Psychiatry Clin Neurosci 2001; 251 (suppl 2): 1166-71.

16 Reed C, Novick D, Gonzalez-Pinto A, Bertsch J, Haro JM. Observational study designs for bipolar disorder - what can they tell us about treatment in acute mania? Prog Neuropsychopharmacol Biol Psychiatry 2009; 33: 715-21.

17 Malig C. The Civil Registration System in Denmark. (IIVRS Technical Paper no. 66). International Institute for Vital Registration and Statistics, 1996.

18 Munk-Jorgensen P, Mortensen PB. The Danish Psychiatric Central Register. Dan Med Bull 1997; 44: 82-4.

19 Danish National Board of Health, L. Medicinal Product Statistics. Danish National Board of Health, 2002 (http://www.laegemiddelstyrelsen.dk)

20 Juel K, Helweg-Larsen K. The Danish registers of causes of death. Dan Med Bull 1999; 46: 354-7.

21 Andersen TF, Madsen M, Jorgensen J, Mellemkjaer L, Olsen JH. The Danish National Hospital Register. A valuable source of data for modern health sciences. Dan Med Bull 1999; 46: 263-8.

22 World Health Organization. Klassifikation af Sygdomme. Systematisk Del. [International Statistical Classification of Diseases and Health Related Problems (10th revision, Danish edn)]. Munksgaard, Copenhagen, 1993.

23 WHO Collaborating Centre for Drug Statistics Methodology. ATC index with DDDs. WHO Collaborating Centre for Drug Statistics Methodology, 2003.

24 Kessing LV, Sondergard L, Kvist K, Andersen PK. Suicide risk in patients treated with lithium. Arch Gen Psychiatry 2005; 62: 860-6.

25 Müller-Oerlinghausen B, Berghofer A, Ahrens B. The antisuicidal and mortality-reducing effect of lithium prophylaxis: consequences for guidelines in clinical psychiatry. Can J Psychiatry 2003; 48: 433-9.

26 Schou M. Lithium treatment at 52. J Affect Disord 2001; 67: 21-32.
27 Schou M. Suicidal behavior and prophylactic lithium treatment of major mood disorders: a review of reviews. Suicide Life Threat Behav 2000; 30 : 289-93.

28 Licht RW, Vestergaard P, Kessing LV, Larsen JK, Thomsen PH. Psychopharmacological treatment with lithium and antiepileptic drugs: suggested guidelines from the Danish Psychiatric Association and the Child and Adolescent Psychiatric Association in Denmark. Acta Psychiatr Scand Supp/ 2003; 419: 1-22.

29 Goodwin GM, Geddes JR. Latest maintenance data on lithium in bipolar disorder. Eur Neuropsychopharmacol 2003; 13 (suppl 2): S51-5.

30 Coryell W. Maintenance treatment in bipolar disorder: a reassessment of lithium as the first choice. Bipolar Disord 2009; 11 (suppl 2): 77-83.

31 Grunze H, Vieta E, Goodwin GM, Bowden C, Licht RW, Moller HJ, et al. The World Federation of Societies of Biological Psychiatry (WFSBP) guidelines for the biological treatment of bipolar disorders: update 2009 on the treatment of acute mania. World J Biol Psychiatry 2009; 10: 85-116.

32 Partonen T, Sihvo S, Lonnqvist JK. Patients excluded from an antidepressant efficacy trial. J Clin Psychiatry 1996; 57: 572-5.

33 Keitner GI, Posternak MA, Ryan CE. How many subjects with major depressive disorder meet eligibility requirements of an antidepressant efficacy trial? J Clin Psychiatry 2003; 64: 1091-3.

34 Zimmerman M, Mattia Jl, Posternak MA. Are subjects in pharmacological treatment trials of depression representative of patients in routine clinical practice? Am J Psychiatry 2002; 159: 469-73.

35 Kleindienst N, Severus WE, Moller HJ, Greil W. Is polarity of recurrence related to serum lithium level in patients with bipolar disorder? Eur Arch Psychiatry Clin Neurosci 2005; 255: 72-4.

36 Licht RW. A new BALANCE in bipolar I disorder. Lancet 2010; 375: 350-2.

37 Cipriani A, Pretty $\mathrm{H}$, Hawton $\mathrm{K}$, Geddes JR. Lithium in the prevention of suicidal behavior and all-cause mortality in patients with mood disorders: a systematic review of randomized trials. Am J Psychiatry 2005; 162: 1805-19.

38 Goodwin FK, Fireman B, Simon GE, Hunkeler EM, Lee J, Revicki D. Suicide risk in bipolar disorder during treatment with lithium and divalproex. JAMA 2003; 290: $1467-73$.

39 Sondergard L, Lopez AG, Andersen PK, Kessing LV. Mood-stabilizing pharmacological treatment in bipolar disorders and risk of suicide. Bipolar Disord 2008; 10: 87-94. 